\title{
Suppression par la lutectomie de l'accroissement simultané de la pression intramammaire et de l'ocytocinémie, induit par l'injection de $\mathrm{PGF}_{2 \alpha}$, chez la brebis
}

\author{
J Labussière 1, MC Lacroix 2, JF Combaud ${ }^{1}$, \\ FA de La Chevalerie ${ }^{1}, \mathrm{P}$ Thomas 1
}

avec la collaboration technique de M Chorho, G Guionneau, $P$ Lambion, $P$ Rolland

\author{
${ }^{1}$ Laboratoire de la traite, INRA, 65 rue de Saint-Brieuc, 35042 Rennes Cedex; \\ 2 Unité d'endocrinologie cellulaire et moléculaire, INRA, 78350 Jouy-en-Josas, France
}

(Reçu le 26 janvier 1989; accepté le 26 octobre 1989)

\begin{abstract}
Résumé - Au $19^{\circ}$ jour de leur gravidité (J19), 7 brebis Lacaune en lactation subissent, soit une lutectomie complète (compensée à partir de ce stade par une complémentation quotidienne de $25 \mathrm{mg}$ de progestérone afin d'assurer la survie embryonnaire; lot $1: 4$ animaux) soit une laparotomie témoin (lot 2 : 3 animaux). L'injection intrajugulaire de $200 \mu \mathrm{g}$ d'un analogue synthétique de $\mathrm{PGF}_{2 \alpha}$ (Dinolytic, Upjohn) ne provoque une augmentation de pression intramammaire (PIM) et l'élévation concomitante de l'ocytocinémie qu'en présence d'un corps jaune, c'est-à-dire sur toutes les brebis des lots 1 et 2 avant J19, et seulement sur celles du lot 2 après ce stade. Ces expériences confirment que c'est le corps jaune, et non les autres compartiments ovariens, qui libère l'ocytocine sous l'action de la prostaglandine $F_{2 \alpha}$.
\end{abstract}

brebis / lutectomie / pression intramammaire / ocytocinémie / prostaglandine $F_{2 \alpha}$

Summary - Luteectomy in the ewe and suppression of the simultaneous rise in intramammary pressure and oxytocinaemia induced by intra-jugular injection of PGF $_{2 \alpha}$. Seven lactating Lacaune ewes underwent either a total luteectomy on day 19 of pregnancy (D19) (Compensated from that stage by a daily progesterone supplementation of $25 \mathrm{mg}$ to ensure embryonic survival; group 1: 4 animals) or a control laparotomy (group 2: 3 animals). Intra-jugular injection of $200 \mu \mathrm{g}$ of a synthetic $P G F_{2 \alpha}$ analogue (Dinolytic, Upjohn) caused an increase in the intramammary pressure (IMP) and a concomitant rise in oxytocinaemia only in the presence of a corpus luteum, ie in all ewes of groups 1 and 2 before $D 19$ and only in those of group 2 after that stage. These experiments confirm that the corpus luteum, and not the other ovarian compartments, releases oxytocin when prostaglandin $F_{2 \alpha}$ is administred.

ewe / luteectomy / intramammary pressure / oxytocinaemia / prostaglandin $F_{2 \alpha}$ 


\section{INTRODUCTION}

L'injection intrajugulaire de PGF $_{2 \alpha}$ ne provoque une éjection du lait que pendant la phase lutéale du cycle sexuel de la brebis (Labussière et al, 1983) et de la vache, (Labussière et al, 1982) ou seulement pendant les 6 et 8 premières semaines de leur gravidité respective (Labussière et al, $1988 \mathrm{a}$ et b). Ces réponses sont abolies par une castration bilatérale, sans qu'il soit possible de les rétablir par une complémentation œstroprogestative (Labussière et al, 1986); on peut donc supposer qu'elles résultent d'une libération d'ocytocine lutéale. Il est en effet maintenant bien établi que le corps jaune de la plupart des espèces sécrète et stocke cette hormone (cf. revue de Wathes et al, 1986) dont la décharge peut être induite par $\mathrm{PGF}_{2 \alpha}$ chez la femme (Gillepsie et al, 1972), la truie (Ellendorff et al, 1979), la chèvre (Cooke et al, 1984), la brebis (Flint et Sheldrick, 1982) ou la vache (Schams et al, 1985).

L'objectif de ce travail est de prouver chez des brebis gestantes que :

- l'augmentation de pression intramammaire consécutive à l'injection de $\mathrm{PGF}_{2 \alpha}$ coïncide avec un accroissement de l'ocytocinémie;

- la lutectomie abolit ces réponses et que l'ocytocine provient bien exclusivement du corps jaune et non d'une autre structure ovarienne, ou de l'embryon qui pourrait sécréter une substance ocytocine-like entre le $14^{e}$ et le $21^{e}$ jour (Lacroix et al, 1988).

\section{MATÉRIEL ET MÉTHODES}

Deux mois environ après la parturition, l'ovulation de 7 brebis Lacaune en lactation (traites à la machine 2 fois par jour) est induite à l'aide d'éponges vaginales en polyuréthane imbibées de $30 \mathrm{mg}$ de FGA (Intervet). Leur retrait, 12 jours plus tard, est accompagné d'une injection intramusculaire de $600 \mathrm{UI}$ de PMSG. L'œstrus (qui apparaît après un délai d'environ $30 \mathrm{~h}$ ) définit le stade Jo. Les brebis sont saillies 2 fois, 12 et $24 \mathrm{~h}$ après cette apparition, et sont alors réparties dans 2 lots :

- le lot $1: 4$ femelles gestantes sont lutectomisées à $\mathrm{J} 19$ et reçoivent à partir de ce stade une complémentation journalière de $25 \mathrm{mg}$ de progestérone (laboratoires Sigma) afin d'assurer la survie des embryons;

- le lot $2: 3$ femelles gestantes subissent à J19 une simple «laparotomie témoin".

L'évolution des variations de pression intramammaire (PIM) provoquées par l'injection intrajugulaire de $2 \mathrm{ml}$ d'une solution de $\mathrm{NaCl} 0,9 \%$ contenant $200 \mu \mathrm{g}$ d'un analogue de $\mathrm{PGF}_{2 \alpha}$ (Dinolytic, Upjohn) est observée de J0 à J28 pour le lot 1 et de Jo à J60 pour le lot 2 . Jusqu'à J28, les enregistrements (réalisés selon la méthode déjà décrite par Labussière et al, 1988a) ont lieu tous les 2 ou 3 jours à 9 h 00 (la traite du matin étant légèrement retardée). Ils sont ensuite espacés de 3 à 6 jours entre J28 et J60 sur le lot 2 qui, seul, continue à être contrôlé.

La progestérone plasmatique est évaluée chaque jour entre J0 et J28 puis chaque jour d'enregistrement de PIM entre J28 et J60. Le sang est prélevé dans l'une des veines jugulaires $(10 \mathrm{ml})$ à l'aide d'un vacutainer hépariné. Les échantillons sont centrifugés $(3000 \mathrm{tr} / \mathrm{min}$ pendant $15 \mathrm{~min})$, le surnageant prélevé $(5 \mathrm{ml}$ environ) et stocké à $-20^{\circ} \mathrm{C}$ en vue du dosage RIA de la progestérone selon la méthode proposée par Saumande et al (1985) et modifiée (immunoprécipitation de $12 \mathrm{~h}$ ) par André (1986, communication personnelle).

L'ocytocinémie est mesurée en même temps que les enregistrements de PIM sur 2 brebis du lot 1 la veille $(J 18)$ et le lendemain $(\mathrm{J} 20)$ de la lutectomie. A chaque contrôle, 3 prélèvements sanguins sont réalisés dans la veine jugulaire 12,7 et 2 min avant l'injection de Dinolytic, puis 5 prélèvements suivent cette administration ( 3 , $8,13,18$ et 28 min après l'injection). Les échantillons sanguins sont traités de la même façon que pour la progestérone et les plasmas conservés au congélateur en vue d'un dosage RIA de l'ocytocine (Geenen et al, 1985).

Les interventions chirurgicales sont toujours pratiquées à $\mathrm{J} 19$ sous anesthésie générale (Labussière et $a l, 1986$ ). Après laparotomie le long de la ligne blanche, les ovaires sont extériorisés: 
- pour le lot 2 : ils sont laissés intacts et replacés immédiatement dans la cavité abdominale qui est refermée;

- pour lot lot 1 : ils sont incisés superficiellement afin de dégager et d'extirper les corps jaunes par compression digitale, puis également replacés dans l'abdomen après cautérisation des afférences vasculaires et suture des lèvres du cratère résultant de l'ablation lutéale.

\section{RÉSULTATS}

La figure 1 indique que :

1) à $J 18$, en présence d'un corps jaune, l'injection intrajugulaire de $200 \mu \mathrm{g}$ de Dinolytic provoque l'augmentation simultanée de la pression intramammaire et de l'ocytocinémie (il importe de remarquer que le taux plasmatique de cette hormone est multiplié par 10 chez la brebis 5747 , possédant 4 corps jaunes et seulement par 2,5 chez la brebis 4801 , qui n'en a qu'un seul);

2) à $\mathrm{J} 20$, après la lutectomie pratiquée à J19, ladministration de prostaglandines $F_{2 \alpha}$ reste sans effet.

II apparaît à la figure 2 qu'après $\mathrm{J} 19$, les accroissements de pression intramammaire ne sont abolis que dans le lot 1 (lutectomisé) alors qu'ils persistent dans le lot 2 (témoin). Dans ce groupe, la diminution progressive des réponses et leur disparition vers la fin du $2^{\mathrm{e}}$ mois de gravidité est en effet comparable à ce qui a été précédemment observé chez des brebis gestantes de la même race (Labussière et al, 1988a).

La supplémentation progestéronique (25 mg/jour) apportée à partir de $\mathrm{J} 19$ aux brebis lutectomisées ne semble pas avoir été suffisante pour compenser l'ablation du corps jaune puisque les taux de progestérone plasmatique restent généralement inférieurs à $2 \mathrm{ng} / \mathrm{ml}$ alors qu'ils atteignaient plus de $4 \mathrm{ng} / \mathrm{ml}$ avant l'intervention et qu'ils se maintiennent à plus de $5 \mathrm{ng} / \mathrm{ml}$ chez les brebis fictivement opérées du lot 2 (fig 3).

\section{DISCUSSION - CONCLUSION}

Ce travail prouve que l'éjection du lait observée après l'administration intraveineuse de $\mathrm{PGF}_{2 \alpha}$ (Labussière et al, 1983, 1986, 1988a) est bien le résultat d'une décharge d'ocytocine, puisque les taux plasmatiques de cette hormone et les niveaux de pression intramammaire s'élèvent simultanément.

L'abolition des 2 types de réponses après l'ablation des corps jaunes plaide en faveur de l'origine lutéale de l'ocytocine. Toutefois, l'origine embryonnaire ne peut être définitivement écartée puisque la faiblesse de notre complémentation progestéronique a pu compromettre la survie des blastocystes, ce qui n'a pu malheureusement être vérifié. Mais il est à notre avis peu probable que les quantités d'ocytocine éventuellement libérées par ceux-ci soient assez importantes pour agir directement au-delà de la sphère utéro-ovarienne. C'est pourquoi, dans l'état actuel de nos connaissances, il est plus plausible d'admettre que la recrudescence des réponses mammaires déjà observées (Labussière et al, 1988) après le $15^{\mathrm{e}}$ jour de gravidité est principalement permise par la reconstitution des stocks lutéaux d'ocytocine (Sheldrick et Flint, 1981a, 1983), même si la synthèse de cette hormone par le corps jaune devient alors faible (Harrisson et al, 1987).

Comme à $\mathrm{J} 16$ ou $\mathrm{J17}$, les brebis "vides" ne présentent aucune éjection du lait lorsqu'on leur administre la même dose (non lutéolytique) de $\mathrm{PGF}_{2 \alpha}$, il est donc envisageable d'utiliser ce test simple, rapide et peu onéreux d'éjection lactée, pour diagnostiquer précocement l'absence de gestation. 

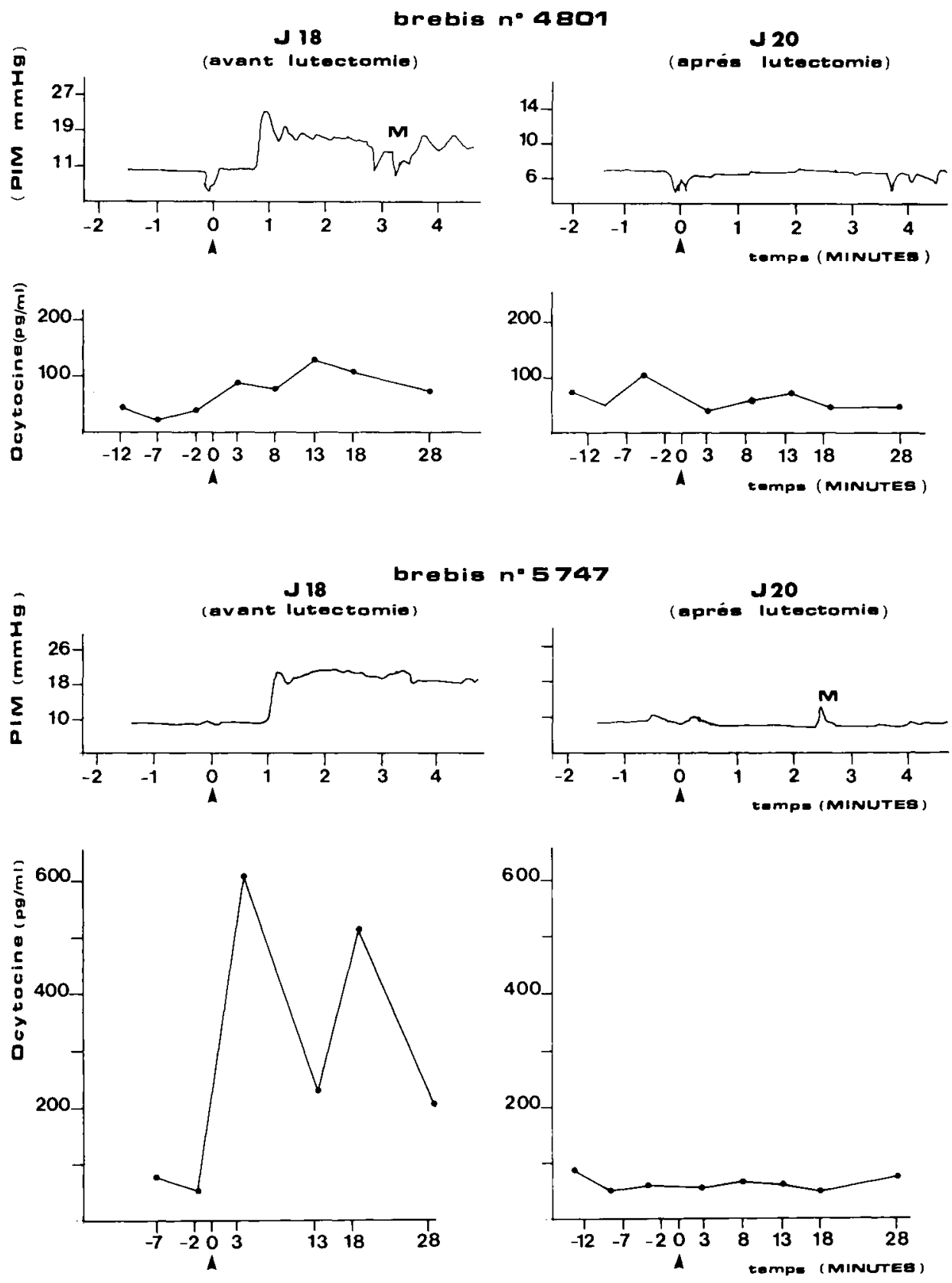

Fig 1. Effets de linjection intrajugulaire de $\mathrm{PGF}_{2 \alpha}$ sur la pression intramammaire et l'ocytocinémie. $\Lambda$ : injection de $200 \mu \mathrm{g}$ de Dinolytic; $\mathrm{M}$ : mouvement de la brebis. 


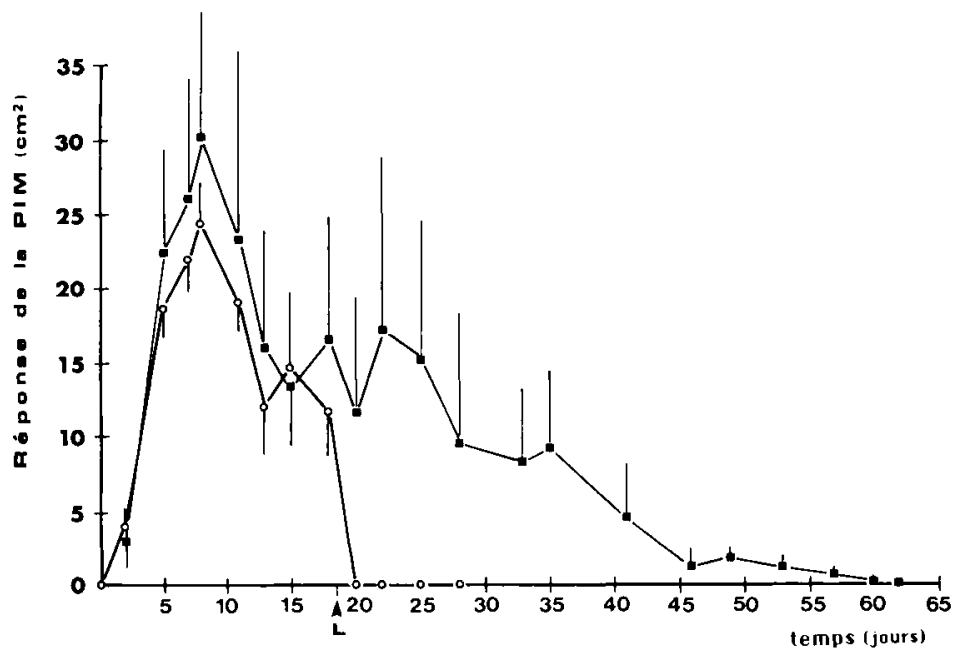

Fig 2. Réponse de la pression intramammaire à l'injection intrajugulaire de $\mathrm{PGF}_{2 \alpha}(200 \mu \mathrm{g}$ de Dinolytic). - brebis gestantes subissant une laparotomie témoin $(n=3) ; 0-0:$ brebis lutectomisées maintenues en gestation par une complémentation journalière de $25 \mathrm{mg}$ de progestérone $(n=4)$. Les valeurs moyennes sont accompagnées de $S \sqrt{n} ; \mathrm{L}$ : stade de la lutectomie (J19) ou de la laparotomie témoin et début de la complémentation progestéronique sur les brebis lutectomisées; le paramètre choisi pour mesurer la réponse de pression intramammaire est la surface située sous la courbe d'enregistrement pendant les 4 premières minutes qui suivent le début de l'accroissement de pression; le jour Jo est celui de l'œstrus.

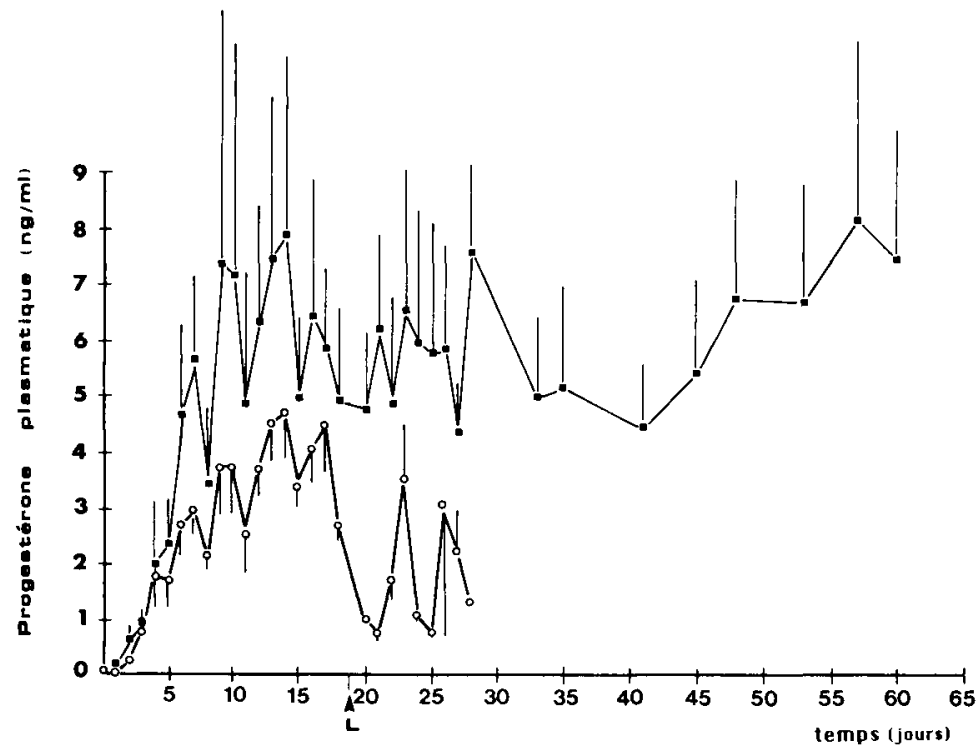

Fig 3. Evolution des taux plasmatiques de progestérone des brebis gestantes subissant une laparotomie témoin $(\square-)$ et des brebis lutectomisées $(0-0)$ maintenues en gestation par une complémentation journalière de $25 \mathrm{mg}$ de progestérone. Les valeurs moyennes sont accompagnées de $S \sqrt{n} ; L$ : stade de la lutectomie (J19) ou de la laparotomie témoin et début de la complémentation progestéronique sur les brebis lutectomisées; le jour J0 est celui de l'œstrus. 


\section{REMERCIEMENTS}

Nous tenons à remercier $M$. D André (INRA, Nouzilly) et M. A Briand (INRA, Rennes) pour l'aide précieuse qu'ils nous ont apportée lors de la réalisation de ce travail.

\section{RÉFÉRENCES}

Cooke RG, Homeida AM, Watkins WB (1984) Simultaneous release of neurophysin and ovarian oxytocin during luteolysis in goat. $J$ Physiol (London) 354

Ellendorff $F$, Forsling $M$, Parvizi $N$, Williams $H$, Taverne M, Smidt D (1979) Plasma oxytocin and vasopressin concentrations in response to prostaglandin injection in the pig. $J R e$ prod Fert 56, 573-577

Flint APF, Sheldrick EL (1982) Ovarian secretion of oxytocin is stimulated by prostaglandin. Nature 297, 587-588

Geenen V, Legros JJ, Hazee-Hagelstein MT, Louis-Hohn S, Leconte-Yerna MJ, Demoulin A, Franchimont $P$ (1985) Release of immunoreactive oxytocin and neurophysin 1 by cultured luteinising bovin granulosa cells. Acta Endocrinol 110, 263-270

Gillepsie A, Brummer HC, Chard T (1972) Oxytocin release by infused prostaglandin. $\mathrm{Br}$ Med J1, 543-544

Harrisson LM, Kenny N, Niswender GD (1987) Progesterone production, $\mathrm{LH}$ receptors and oxytocin secretion by ovine luteal cell types on days 6,10 and 15 of the cestrous cycle and day 25 of pregnancy. $J$ Reprod Fert 79 , 539-548

Labussière J, Philibert C, Combaud JF, Dotchewski $D$ (1982) Etude de l'efficacité de $\mathrm{PGF}_{2 \alpha}$ sur l'éjection du lait au cours du cycle sexuel de la vache. Reprod Nutr Dev 22, 4963

Labussiere J, Philibert C, Dotchewski D, Combaud JF, de La Chevalerie FA, Bernabé J (1983) Effets des prostaglandines $F_{2 \alpha}$ sur l'éjection du lait de la brebis. Variations au cours du cycle sexuel. Proceedings of the
IIIrd Symposium International "Ordeño Mecanico de Pequeños Rumiñantes". Sever Cuesta, Valladolid, Espagne, 64-80

Labussière J, Eyi Ngui V, Combaud JF (1986) Effets des prostaglandines $\mathrm{PGF}_{2 \alpha}$ sur l'éjection du lait de la brebis. Conséquences de l'ovariectomie accompagnée ou non d'une complémentation œstroprogestative. Reprod Nutr Dev 26, 933-942

Labussière J, Combaud JF, de La Chevalerie FA (1988a) PGF $_{2 \alpha}$ induced milk ejection in ewes having cyclic of pregnant corpora lutea. Reprod Nutr Dev 28, 541-552

Labussière J, Thomas $P$, Combaud JF, de La Chevalerie FA (1988b) Ejection du lait induite par $\mathrm{PGF}_{2 \alpha}$ pendant les 2 premiers mois qui suivent l'insémination des vaches. Application à un diagnostic de gravidité. Reprod Nutr Dev 28, 899-907

Lacroix MC, Charpigny G, Reinaud P (1988) Is oxytocin of conceptus origin involved in inhibition of luteal regression in early pregnancy in ewes? J Endocrinol 118, R17-R20

Saumande J, Tamboura D, Chupin D (1985) Changes in milk and plasma concentration of progesterone in cows after treatment to induce superovulation and their relationships with number of ovulation and of embryos collected. Theriogenology 23, 719-731

Schams D, Schallenberger E, Legros JJ (1985) Evidence for the secretion of immunoreactive neurophysin 1 in addition to oxytocin from the ovary in cattle. J Reprod Fert 73, 165-171

Sheldrick EL, Flint APF (1981) Circulating concentrations of oxytocin during the cestrous cycle and early pregnancy in sheep. Prostaglandins 22, 631-636

Sheldrick EL, Flint APF (1983) Luteal concentrations of oxytocin decline during early pregnancy in the ewe. J Reprod Fert 68, 477-480

Wathes DC, Swann RW (1982) Is oxytocin an ovarian hormone? Nature (London) 297, 225227

Wathes DC, Swann RW, Porter DG, Pickering BT (1986) Oxytocin as an ovarian hormone. In: Current Topics in Neuroendocrinology. Vol. 6 : Neurobiology of Oxytocin. Ganten D, 6900 Heidelberg, RFA, 129-152 